\title{
Thermodynamic Explanation for the Cosmic Ubiquity of Organic Pigments
}

\author{
Karo Michaelian ${ }^{1^{*}}$ and Aleksandar Simeonov ${ }^{2}$ \\ ${ }^{1}$ Instituto de Física, Universidad Nacional Autonoma de México, Mexico. \\ ${ }^{2}$ Institute of Biology, Saints Cyril and Methodius University of Skopje, Macedonia
}

"Corresponding author: Karo Michaelian, Instituto de Física, Universidad Nacional Autonoma de México, Circuito de la Investigación Cientifica, Cuidad Universitaria, Mexico, Tel: 525562225001; Email: karo@fisica.unam.mx

Received date: October 21, 2016; Accepted date: February 2, 2017; Published date: February 8, 2017

Copyright: (C) 2017 Michaelian K, et al. This is an open-access article distributed under the terms of the Creative Commons Attribution License, which permits unrestricted use, distribution, and reproduction in any medium, provided the original author and source are credited.

\begin{abstract}
There is solid evidence for the occurrence of large amounts of organic material in the cosmos, particularly in the form of aromatic compounds. These molecules can be found on the surface of Earth and Mars, in the atmospheres of the larger planets and on many of their satellites, on asteroids, comets, meteorites, the atmospheres of red giant stars, interstellar nebulae, and in the spiral arms of galaxies. Many of these environments are expected to be of low temperature and pressure, implying that the Gibb's free energy for the formation of these complex molecules should be positive and large, suggesting that their existence could only be attributed to non-equilibrium thermodynamic processes. In this article we first review the evidence for the abundance of these molecules in the cosmos and then describe how the ubiquity can be explained from within the framework of non-equilibrium thermodynamics on the basis of the catalytic properties of these pigment molecules in dissipating photons of the ultraviolet and visible emission spectra of neighboring stars, leading to greater local entropy production. A relation between the maximum wavelength of absorption of these organic pigments and the corresponding stellar photon environment provides a guide to determining which aromatic compounds are most probable in a given stellar neighborhood, a postulate that can be verified on Earth. It is suggested that at least some of the baryonic dark matter may be associated with these molecules which emit in the extreme infrared with many, but weak, emission lines, thus so far escaping detection. This thermodynamic explanation for the ubiquity of these organic molecules also has relevance to the possibility of life, both as we know it, and as we may not know it, throughout the universe.
\end{abstract}

Keywords: Astrobiology; Cosmic organic material; UV Photochemistry; Entropy production; Aromatic Infrared Bands (AIBs)

\section{Introduction}

In 1937 , the detection of the methylidyne $(\mathrm{CH})$ radical in early type stars $(\mathrm{O}, \mathrm{B}$ and $\mathrm{A}$ stars) $[1,2]$ ushered in an era of astrochemical discoveries. It was simultaneously the first molecule and the first organic compound to be ever discovered in extrasolar space.

An upsurge of molecular discoveries followed during the sixties and seventies, with the discovery of ammonia [3], water [4], formaldehyde [5], methanol [6], and carbon monoxide [7]. Of great importance to the question of the origin of life was the detection of N-containing organics like hydrogen cyanide [8], methylamine [9], and cyanamide [10], since it had been shown that $\mathrm{HCN}$ in the presence of water and a free energy source is sufficient to produce the DNA purines and pyrimidines [11-14]. It therefore came as no surprise when the nucleobases were discovered in carbonaceous meteorites, along with some unusual and terrestrially rare analogs (purine, 2,6diaminopurine, and 6,8-diaminopurine), indicative of their extraterrestrial origin $[15,16]$. Another important discovery was that of interstellar acetylene [17] a precursor molecule to benzene and hence other aromatic and polyaromatic organic molecules [18].

As of 2016, approximately 200 gas-phase molecular species, most of them organic, have been recorded as common constituents of the interstellar medium and circumstellar shells $[19,20]$.
Pivotal to the search and the accumulation of data has been the development of millimeter-wave and infrared spectroscopy which, through the detection of the stretching and bending vibrational modes of covalent bonds, as well as rotational transitions, has confirmed the existence of a vast array of these molecular types in space [21].

The large abundance of the element carbon (fourth most abundant element in the universe after $\mathrm{H}, \mathrm{He}$ and $\mathrm{O}$ [22]), its unique covalent bonding flexibility, and the abovementioned pioneering discoveries of low molecular weight organics which can act as chemical precursors to other molecules, have spurred the search for larger, more complex organics in space.

In some unconventional and truly startling comparisons, Fred Hoyle and Chandra Wickramasinghe, the founders of the theory of panspermia, showed how the extinction spectra over the ultraviolet and visible of interstellar nebulae can be fitted well by the absorption spectra of freeze dried algae and bacteria [23]. More recent higher resolution data refute the quality of the fit [24], but there can be little doubt that the nature and complexity of the absorbing material in the interstellar clouds must be somehow analogous to that of the biological macromolecules. The Extended Red Emission (ERE) feature, for example, which is a photoluminescence process powered by UV photons with a broad peak emission wavelength around 650-800 nm [25], most closely parallels the optical behavior of chlorophyll and other porphyrinic bio-pigments, that have absorbance in the near-UV/ blue and fluorescence in the red [26].

Moreover, the ERE is only one of a family of universal spectroscopic features, which, although not yet fully explained, are typically regarded 
as spectral "fingerprints" of complex organic compounds throughout the cosmos. These include: the Unidentified Infrared Emission (UIE) bands, the Diffuse Interstellar absorption Bands (DIBs), the $217 \mathrm{~nm}$ feature, and the 21 and 30 micron emission features [27].

In particular, the UIE bands, which are emission bands originating from numerous galactic and extragalactic astronomical environments, have traditionally been considered as the spectral signatures of polycyclic aromatic hydrocarbons (PAHs) [28,29], although newer spectroscopic analyses [30,31] revive earlier proposals [32] for even more complex chemical structure of the carriers of these bands; Mixed Aromatic/Aliphatic Organic Nanoparticles (MAONs), very reminiscent of the complex macromolecular structure of kerogen and coal on Earth, and the Insoluble Organic Matter (IOM) of carbonaceous chondrites [33].

There is thus unequivocal evidence for the formation of large and complex organic molecules throughout the universe. However, these complex molecules are prone to photo-ionization and destruction by charged particles, x-rays, and the same high-frequency UV photons that probably helped formed them. Therefore, their ubiquity, and thus the probable existence of life as we know it in the cosmos, requires an explanation that goes beyond near-equilibrium reaction pathways of formation. That these molecules are the result of non-equilibrium thermodynamic processes can be seen from the fact that their occurrence is dependent upon a continuous flow of UV photons from the neighboring star. However, this paper goes further to show that the ubiquity of organic pigments throughout the cosmos, wherever there exists UV light interacting with material, is a result of the non-linear, non-equilibrium nature of the photochemical process leading to the organic molecules. Specifically, it is shown that these molecules can be considered as microscopic self-organized dissipative structures which form and proliferate to dissipate the generalized thermodynamic force; the stellar photon potential formed between the high energy photon outflow of a neighboring star and the low energy (microwave) photon cosmic background of space. In other words, these pigment molecules owe their existence and proliferation to the entropy production that they perform by dissipating high energy photons into heat.

\section{Evidence for the cosmic ubiquity of organic pigments}

Infrared observations by Gillett et al. [34] and Russell et al. [35,36] revealed that the IR spectra of bright astronomical objects with associated gas and dust particles, such as HII regions and planetary nebulae, are dominated by strong, broad, and resolved emission bands. Since the carrier of the bands remained unidentified for almost a decade, they were dubbed the Unidentified Infrared Emission (UIE or UIR) bands. The fact that their intensity showed a direct correlation with carbon abundance within the source naturally implied a carbonbased carrier.

Knacke [37] and Duley and Williams [38] first suggested aromatic carriers, since the bands peak at wavelengths corresponding to the stretching and bending modes of various $\mathrm{CH}$ and $\mathrm{CC}$ bonds in aromatic hydrocarbons, so they also became known as the Aromatic Infrared Bands (AIB or AIR).

With the launch of the Infrared Space Observatory (ISO) mission in 1995 the UIE features were observed with better spectral resolution, covering a broader spectral range, and in a larger sample of sources. They were found to dominate the mid-IR emission of almost all astronomical objects except for asymptotic giant branch (AGB) stars and deeply embedded young stellar objects [39]. It was estimated that around $20-30 \%$ of the infrared radiation of our galaxy is being emitted in these infrared emission bands [40], and up to $20 \%$ of the total radiation output of distant starburst galaxies [41,42], indicating that their carrier must be an extremely common and abundant cosmic material.

Solar System objects, such as Interplanetary Dust Particles (IDPs), carbonaceous meteorites, micrometeorites, Martian rocks [43], comets [44], Titan's upper atmosphere [45] are also known to display these infrared features.

A wide variety of hydrocarbon and carbonaceous materials comprising aromatic units have been proposed as possible carriers of the AIB bands, including; Hydrogenated Amorphous Carbon (HAC), Quenched Carbonaceous Composites (QCC), Polycyclic Aromatic Hydrocarbons (PAHs), soot and carbon nanoparticles, fullerenes and fullerene-like particles, coal and kerogen, petroleum fractions etc. [46]. Among all these models, the PAH hypothesis developed by Leger and Puget [47] and Allamandola et al. [28,29] has gained most attention, therefore the UIE bands are usually referred to in the literature simply as the PAH bands.

Polycyclic Aromatic Hydrocarbons (PAHs) are benzene rings of $\mathrm{sp}^{2}$ hybridized $\mathrm{C}$-atoms linked to each other in a plane, with $\mathrm{H}$ atoms or other functional groups saturating the outer bonds of peripheral Catoms. Electron delocalization over their entire carbon skeleton makes them exceptionally stable because photonic excitation energy quickly becomes dispersed over the entire molecule before it can break a single bond.

According to the most accepted hypothesis, the infrared bands are attributed to the vibrational relaxation of PAH molecules containing around $50 \mathrm{C}$-atoms on average, which are stochastically heated to high temperatures $(\sim 1000 \mathrm{~K})$ by the absorption of individual high-energy UV photons $[48,49]$. In the low density environment of the interstellar medium, where collisional de-excitation is not possible, these UVpumped, gas-phases PAH molecules undergo spontaneous deexcitation via infrared fluorescence which gives the AIB features.

While the hypothesis requires strong UV radiation field to excite the PAH molecules (PAHs absorb strongly only in the UV), Uchida et al. [50,51] observed UIE features in the colder radiation environments of reflection nebulae, implying that other types of molecules may be responsible at least for some part of the emissions.

In support of this supposition is the fact that the PAH model is unable to explain the appearance of some aliphatic emission features at 3.4, 6.9 and $7.2 \mu \mathrm{m}$ and the superimposition of the AIBs upon broad and strong emission plateau features at 6-9, 10-15, and 15-20 $\mu \mathrm{m}$, which were shown to be of aliphatic nature $[52,53]$.

Kwok and Zhang [30,31] made spectral decomposition analyses of archival spectroscopic observations of the UIE bands and showed that the data are most consistent not with pure, free-flying PAH molecules, but with solid organic nanoparticles that have a mixed aromaticaliphatic $\left(\mathrm{sp}^{2}-\mathrm{sp}^{3}\right)$ structure. They called this new model MAON (Mixed Aromatic/Aliphatic Organic Nanoparticle). The proposed MAON emission carriers are quite different from the pure hydrocarbon, planar, gas-phase, relatively small molecular $(\sim 50 \mathrm{C}$ atoms) PAHs, where the structure, no matter how large, is regular with repeatable patterns. MAONs are amorphous solids of hundreds or thousands of C-atoms and impurity elements such as $\mathrm{O}, \mathrm{N}$, and $\mathrm{S}$, with a disorganized, three-dimensional macromolecular structure 
comprised of aromatic and aliphatic units, each with variable sizes and random orientations.

Since the late 1980's Papoular et al. have argued that the observed spectral properties of the UIE bands most closely resemble those of coal and kerogen $[32,54,55]$, which are actually organic, amorphous, macromolecular materials with randomly oriented clusters of aromatic rings linked by long aliphatic chains, with $\mathrm{O}, \mathrm{N}, \mathrm{S}$ functional groups and heterocycles in their structure. This complex macromolecular structure is also very similar to the structure of the Insoluble Organic Matter (IOM) found in the Murchison meteorite and identified in laboratory analyses by Derenne and Robert [56]. More than $70 \%$ of the organic matter in carbonaceous chondrites, such as the Murchison, Murray, Tagish Lake, and Orgueil meteorites is in this form of insoluble amorphous solids $[57,58]$. The structure of the IOM in all these meteorites shows remarkable resemblance [59], while isotopic ratios point to their interstellar origin $[60,61]$.

It is interesting that in the UV-poor environment of protoplanetary nebulae (PPN), the aliphatic chains of these UIE emitters constitute a significantly larger percentage of the entire macromolecular structure when compared to the UV-intense regions of Planetary Nebulae (PN) where the aromatic component is predominant $[53,62]$. For example, in the PPN phase of stellar evolution, the 3.4 and $6.9 \mu \mathrm{m}$ aliphatic emission features are as strong as the 3.3, 6.2, 7.7, and $11.3 \mu \mathrm{m}$ aromatic emission features, but as the star evolves to the PN stage (a process of less than few thousand years), the aromatic features become considerably stronger [62]. This phenomenon has been explained as the result of photochemistry, where the onset of UV radiation modifies the aliphatic side groups through cyclizations and isomerizations, and transforms them into aromatic ring structures, making the macromolecule more aromatic $[63,64]$.

Much of the diverse organic material found on the surface of Earth is in the form of pigments that absorb strongly across the UV and visible wavelength region and dissipate this energy rapidly into heat (vibrational energy) which is then further dispersed over the vibrational modes of the surrounding solvent water molecules [65-68]. This is true of DNA and RNA which absorb and dissipate strongly from 220-280 nm, for the aromatic amino acids tyrosine, tryptophan, and phenylalanine which absorb strongly from $220-300 \mathrm{~nm}$, for the mycosporine-like amino acids and scytonemin which absorb over 300-500 nm, and for the other common pigments of phototropic organisms; chlorophylls, phycobilins, carotenoids, anthocyanins, which absorb from the visible to the infrared.

It is an intriguing fact that most of the fundamental molecules of life (those found in all three domains of life) are also pigments which absorb in the UV-C and have chemical affinity to RNA and DNA. Through Förster-type energy transfer, as well as through the coupling of vibronic modes [69], these molecules can also use RNA and DNA as acceptor quencher molecules to dissipate extremely rapidly (through a conical intersection) the absorbed photon excitation energy into heat $[67,68]$.

Planet Earth has for a long time been considered as the only body in the solar system that contains organic matter, usually attributed to the activities of life. The conventional picture for the chemical composition of other solar system bodies (planets, satellites, asteroids, comets, and dust particles) is that they are entirely made up of metals, minerals, ices and gases, with either no or only traces of organics. However, with the development of more sophisticated technologies like sending spacecrafts to solar system bodies for close spectroscopic observation and direct sample collection, organic substances are increasingly being recognized as a major component of planetary chemistry.

One of the first hints of the presence of organic matter on the surface of asteroids came from their deep red colors and low albedos (0.01-0.15). Such intense colors and low albedos are difficult to explain considering only inorganic minerals and ices but can easily be explained by organic pigments with a complex kerogen-like macromolecular composition [70]. Roush and Cruikshank [71] have noted that terrestrial dark pigmented organic solids like tar sands, asphaltite, pyrobitumen, and kerite, which all have complex aromatic/ aliphatic composition, have very low albedos and dark reddish-brown colors, reminiscent of asteroids.

Saturn's largest moon Titan, the only known natural satellite to have a dense atmosphere, has attracted much attention since the CassiniHuygens unmanned spacecraft, which arrived there in 2004, revealed that Titan's atmosphere contains haze-like solid particles that are the result of condensation of organics. The observations support the hypothesis that methane and nitrogen molecules excited by UV photons react to form polymeric hydrogenated carbon-nitride compounds, called tholins, which give the distinctive thick layer of orange-brown haze in Titan's lower stratosphere [72,73]. Cassini RADAR observations found that these organic nanoparticles condense on the surface of sand grains that are blown into longitudinal darkcolored dunes by winds. Most interestingly, some of these organic nanoparticles end up dissolved in the numerous lakes and rivers of liquid methane and ethane in Titan's polar regions, which exhibit active liquid-gas phase cycling, similar to the water cycle on Earth, although at much lower temperature [74].

The Cassini spacecraft has also found evidence of mixed aromatic/ aliphatic hydrocarbons on other Saturnian satellites: Iapetus, Phoebe and Hyperion [75-77]. Iapetus is the third largest satellite of Saturn, locked in synchronous rotation about the planet, with the leading hemisphere and sides of dark brown color and low albedo (0.03-0.05), and most of the trailing hemisphere and poles of bright color and high albedo (0.5-0.6) [78]. This difference in coloring between the two hemispheres is striking. Temperatures on the dark region's surface reach about $129 \mathrm{~K}$ at the equator, while the bright surfaces reach only about $113 \mathrm{~K}$ due to less sunlight absorption [79]. The low-albedo material exhibits mainly an aromatic hydrocarbon signature [80].

Trans-Neptunian Objects (TNO) are a group of minor bodies that orbit the Sun in the outer solar system beyond the planet Neptune. Photometric observations in the visible have found the colors of some of these objects to be intensely red [81], which can be an indication of the presence of organic material on their surface.

Exceptionally rich in organic content and organic diversity are comets [82]. They represent agglomerates of rocky debris, frozen gases and ices, and are likely the most primitive bodies in the solar system [83]. Dust samples from the coma of comet Wild 2 were collected by the Stardust spacecraft and returned to Earth in 2006. The samples were analyzed with various techniques, giving evidence of rich organic content, but mostly in the form of aromatic-aliphatic macromolecules similar to the IOM of meteorites but with higher $\mathrm{N}$ and $\mathrm{O}$ content. Of particular significance is that the aromatic compounds are not pure hydrocarbons but have a very high $\mathrm{N}$ content, where $\mathrm{N}$ is incorporated predominantly in the form of aromatic nitriles $(\mathrm{R}-\mathrm{C} \equiv \mathrm{N})$; a fact of great astrobiological implication as aromatic nitriles are precursory to many organic pigments and other fundamental biological molecules, and 
comets are likely to have contributed to Earth's prebiotic chemical inventory $[44,84]$.

In the following section, we will show how, under an imposed UV-C light flux from nearby stars, non-equilibrium thermodynamic principles based on the general evolutionary criterion of Classical Irreversible Thermodynamics, leading to increases in entropy production through UV-photon dissipation, would indicate the formation, proliferation, and aromatization of these organic compounds and thus explain their ubiquity in the cosmos.

\section{A non-equilibrium thermodynamic explanation for organic pigment ubiquity}

It is thus now abundantly clear that a large class of pigment molecules exist throughout the cosmos in the form of tholins, polyaromatic hydrocarbons (PAHs) and/or Mixed Aromatic/Aliphatic Organic Nanoparticles (MAONs) and that these systems are absorbing light in the UV and visible spectrums and dissipating it into infrared emission bands. The dissipative process in which a part of the incident solar spectrum is absorbed and dissipated into heat by organic pigment molecules at the Earth's surface is by far the most important entropy producing process occurring on Earth. Similarly, the interstellar reddening due to photon dissipation by the interstellar PAHs and/or MAONs is probably the most important entropy producing process occurring in the cosmos after nuclear fusion and the expansion of the universe.

It will now be shown that the formation of these pigment molecules can be viewed as microscopic self-organization of material (microscopic dissipative structuring) in response to the imposed UV photon potential from nearby stars and that pigment proliferation to well beyond expected equilibrium concentrations follows from the same non-linear, non-equilibrium thermodynamic principles. Our thermodynamic analysis will follow that of Prigogine [85] for a set of purely autocatalytic chemical reactions, the difference being that instead of dissipating chemical potentials, our cosmic pigment system will be dissipating photochemical potentials related to the difference in the photon pressure of the relevant stellar spectra and that of the cosmic microwave background spectrum.

Advantages for microscopic dissipative structuring utilizing photochemical reactions over normal thermally induced chemical reactions occurring in the electronic ground state are various [86];

1. Reactions may occur that are very endothermic in the ground state since the absorbed photon donates its energy to the molecule. For example, the energy of a photon of $260 \mathrm{~nm}$ (UV-C) is $4.77 \mathrm{eV}$ or $110 \mathrm{Kcal} / \mathrm{mol}$.

2. In the electronic excited state antibonding orbitals are occupied and this may allow reactions which are not possible for electronic reasons in the ground state.

3. Photochemical reactions can involve singlet and triplet states Thermal reactions usually only involve singlet states. Therefore, photochemical reaction intermediates may be formed which are not accessible under thermal conditions.

The coupled photochemical dissipative process involving UV-C light dissipation and cosmic pigment production, which drives pigment proliferation, is the transformation of a black-body spectrum at a high temperature, that of the surface of a nearby star (e.g., our sun at 5760 $\mathrm{K})$ into an intermediate black-body spectrum at a lower temperature (that of the vibrational spectrum of the low molecular weight precursor molecules of the pigments) after absorbing UV-C photons, and finally the dissipation to a black-body spectrum at a temperature of the infrared emission bands. Besides this photon dissipation process, we also have a "parasitic" photochemical reaction producing pigments from the excited precursor molecules. These pigments then act as catalysts for the dissipation of the incident stellar spectrum into the infrared emission spectrum, thereby making the global dissipation process autocatalytic and this brings the non-linearity into the nonequilibrium thermodynamic process. It is this nonlinearity that leads to pigment proliferation just as it does to catalyst proliferation in a purely autocatalytic chemical reaction process.

We will show that in the thermodynamic stationary state, the concentration of the pigment molecules can grow many orders of magnitude greater than what would be expected if the system were closer to equilibrium or if the pigment were not an effective catalyst in the photon dissipation. This is the same reasoning which explains the concentration of a product catalyst in a normal autocatalytic chemical reaction growing many orders of magnitude larger than its expected equilibrium concentration due to its involvement in the dissipation of the imposed chemical potential [85].

Photons carry momentum, and thus for a collection of photons in equilibrium (a black-body spectrum), a unique equilibrium pressure can be defined which is found to go as the fourth power of the temperature. It is assumed here that the rate of energy conversion between spectra will be linearly proportional to the difference of the photon pressures of the different spectra. This is similar to assuming that the rate of a chemical reaction is proportional to the difference in the concentrations of the reactant and product (with equilibrium and rate constants set equal to one), or that the flow of material is proportional to the gas pressure difference, or that the flow of electrical current is proportional to the difference in electrical potential. These latter two linear relationships for transport processes are valid when the mean free paths (between scattering events) of the particles are small with respect to the size of the system, i.e. that the system attains local equilibrium, while for the former chemical reactions (or photon dissipation), it is only required that the reactants and products (or photons) retain a Maxwell-Boltzmann (or Boltzmann) distribution of the velocities (energies) of the particles (or photons) involved.

There are then four relevant photon pressures, 1) $\mathrm{P}_{\mathrm{S}}$ corresponding to the stellar photon spectrum arriving at the site of the precursor molecules, an approximate black-body spectrum with a temperature equal to that of the surface of the local star, 2) $\mathrm{P}_{\mathrm{I}}$ corresponding to the vibrational, assumed black-body, temperature of the precursor molecules after absorbing the energy of a UV-C photon and then electronic de-excitation through a conical intersection, converting electronic energy into vibrational energy, and 3) $\mathrm{P}_{\mathrm{E}}$ the photon spectrum of the infrared emission bands, 4) $\mathrm{P}_{\mathrm{p}}$ corresponding to the spectrum emitted by the produced pigment which will be dependent on the local concentration of the pigments and the energy diverted into forming their covalent bond structures.

Although using a black-body assumption for these spectra is in error since the incident radiation from the star has particular directionality properties and only approximates a black-body spectrum, the purpose of the calculation here is to show qualitatively how the proliferation of organic pigments in the cosmos can be explained from a non-linear irreversible thermodynamic analysis of the photon dissipation process. A more accurate derivation of this would employ Planck's formula for the entropy of an arbitrary beam of photons. 
Employing the formalism of classical irreversible thermodynamics [85], the entropy production is a product of generalized forces, $\mathrm{X}$, times generalized flows, J,

$$
\mathrm{P}=\mathrm{d}_{\mathrm{i}} \mathrm{S} / \mathrm{dt}=\sum_{\mathrm{k}} \mathrm{J}_{\mathrm{k}} \mathrm{X}_{\mathrm{k}} \geq 0
$$

Where the sum is over all the irreversible processes occurring within the system. As an example, for a chemical reaction, the generalized force $\mathrm{X}$ is the affinity over the temperature $\mathrm{A} / \mathrm{T}$, where the affinity $\mathrm{A}$ for the reaction is equal to the stoichiometric coefficients of the reactants multiplied by the chemical potentials minus the same of the products. The chemical potentials, in turn, depend on the concentrations of the reactants and products and the affinity can be derived from the Gibb's free energy differences of the reactant and product states. The generalized flow J for the irreversible process of a chemical reaction is the rate of the reaction.

For our photon dissipation irreversible process to be considered here, the generalized forces are the photon spectrum affinities determined from the photon pressures, and the generalized flows are the flows of energy which are transformed from the incoming short wavelength spectrum to the emitted long wavelength spectra.

The change of the entropy production $\mathrm{dP}$ can be decomposed into two parts, one related to the change of forces and the other to the change of flows [85],

$$
\mathrm{dP}=\mathrm{d}_{\mathrm{X}} \mathrm{P}+\mathrm{d}_{\mathrm{J}} \mathrm{P}=\sum_{\mathrm{k}} \mathrm{J}_{\mathrm{k}} \mathrm{dX}_{\mathrm{k}}+\sum_{\mathrm{k}} \mathrm{X}_{\mathrm{k}} \mathrm{dJ}_{\mathrm{k}}
$$

Where the sums are over all irreversible processes occurring within the system.

In the whole domain of the validity of thermodynamics of irreversible processes, and under the condition of constant external constraints, the contribution of the time change of the forces to the entropy production is negative or zero,

$$
\mathrm{d}_{\mathrm{X}} \mathrm{P} \leq 0 .
$$

This is known as the general (or universal) evolutionary criterion, or the Glansdorff-Prigogine criterion, since it was first established by Glansdorff and Prigogine [87] (see also Prigogine [85]). The general evolutionary criterion is the most general result that has thus far been obtained from Classical Irreversible Thermodynamics. For systems with constant external constraints (for example, a constant flux of UV photons from a neighboring star), the system will eventually come to a thermodynamic stationary state in which case [85],

$$
\mathrm{d}_{\mathrm{X}} \mathrm{P}=0 \text {. }
$$

With this background in classical irreversible thermodynamics, we can now analyze our particular irreversible processes consisting of the conversion of energy through different photon spectra. First, the incident flow of energy of the photon spectrum coming from the surface of the star $I_{S}(\lambda)$, which can be approximated by a black-body spectrum for convenience, $I_{S}\left(T_{S}\right)$, where $T_{S}$ is the temperature of the surface of the star (say $5760 \mathrm{~K}$, for a G-type star like our sun), is converted, by absorption on precursor molecules followed by either a photochemical reaction leading to a pigment molecule, or by the dissipation directly into heat giving an intermediate spectrum of intermediate temperature (also assumed to be black-body), $\mathrm{I}_{\mathrm{I}}\left(\mathrm{T}_{\mathrm{I}}\right)$, with say $\mathrm{T}_{\mathrm{I}}=1000 \mathrm{~K}$ (corresponding roughly to the vibrational temperature of a molecule of 50 atoms or so after absorbing a single UV-C photon). This spectrum is then converted through interaction of the molecule with its gas or solid MAON surroundings into the red-shifted photon spectrum of the infrared emission bands $\mathrm{I}_{\mathrm{E}}\left(\mathrm{T}_{\mathrm{E}}\right)$, with say $\mathrm{T}_{\mathrm{E}}=287 \mathrm{~K}$.
Note that in the very low density environment of space, aggregation of individual molecules into large structures like MAONs would be thermodynamically selected since this would allow distribution of the incident photon energy over more microscopic degrees of freedom, or, in other words, emission at lower temperatures, giving greater entropy production. Small isolated molecules in a vacuum would have to fluoresce at high frequencies in order to dissipate the excitation energy of a single UV-C photon, leading to less entropy production.

This process can be equivalently looked at as one in which, at the intermediate temperatures of the vibrationally excited precursor molecules, a photochemical reaction may take place in which some of the free energy of the vibrationally excited molecules is diverted into the production of organic pigments leading to an emitted photon spectrum of the newly formed pigments which is dependent on the concentration $\mathrm{C}$ of the pigments and how much free energy went into forming the relevant covalent bonding and is also assumed to be characterized by a black-body spectrum of temperature $T_{p}$.

These newly formed pigments themselves act as catalysts for the overall dissipation process of $\mathrm{I}_{\mathrm{S}}\left(\mathrm{T}_{\mathrm{S}}\right) \rightarrow \mathrm{I}_{\mathrm{E}}\left(\mathrm{T}_{\mathrm{E}}\right)$, and can be even more catalytic by, for example, providing local heat of dissipation to increase the probability of the photochemical reaction leading to a pigment $\mathrm{I}_{\mathrm{I}}\left(\mathrm{T}_{\mathrm{I}}\right) \rightarrow \mathrm{I}_{\mathrm{p}}\left(\mathrm{T}_{\mathrm{p}}\right)$. Photochemical reactions are temperature dependent, just like normal chemical reactions. These two effects make the dissipation process auto-catalytic. A schematic diagram for this autocatalytic photochemical process can be given as follows;

$$
\begin{array}{cc}
\text { Is (Ts) } \stackrel{1}{\rightarrow} & I_{I}\left(T_{I}\right) \stackrel{2}{\rightarrow} \mathrm{I}_{\mathrm{E}}\left(\mathrm{T}_{\mathrm{E}}\right) \\
& \mathbb{\Downarrow}^{3} \\
& I_{P}\left(T_{P}\right)
\end{array}
$$

Since the system is far from equilibrium, the backward rate constants for the spectra conversions can be considered as being essentially zero, which is also the case for the photochemical reaction leading to the pigments (reaction 3). Note, however, that if a high energy photon can produce a pigment, a similar photon could also destroy it. There is escape from this predicament if the pigment is fortuitously produced with a conical intersection allowing rapid nonradiative dissipation, thereby neutralizing the destructive potential of the UV-C photons. Pigments produced without a conical intersection, but having chemical affinity to a molecule that has one, so that the pair could operate in the donor-quencher mode, would also be spared from the destructive potential of the UV-C photons. Such a selection process would tend to build up pigments with conical intersections or pigments with chemical affinity to those that have one, forming complexes like the MAON structures.

Since the photon spectra are all considered to be black-body, they are completely characterized by one variable which is the black-body temperature $\mathrm{T}$, or equivalently by the photon pressure $\mathrm{P}$, which goes as $\mathrm{T}^{4}$. Thus, the conversion of the energy through the different spectra and the energy flow into the formation of the pigments can be characterized, in a first approximation, by the pressures corresponding to the assumed black-body spectra. We can therefore rewrite equation (5) alternatively in terms of pressures; 


$$
\begin{gathered}
P_{S} \stackrel{1}{\rightarrow} P_{I} \stackrel{2}{\rightarrow} P_{E} \\
\mathbb{\Downarrow}^{3} \\
\mathrm{P}_{\mathrm{P}}
\end{gathered}
$$

where, as mentioned above, $\mathrm{P}_{\mathrm{P}}$ is the photon "pressure" related to the black-body temperature of the pigments after their production which is related to their concentration or free energy that went into their formation. It is also assumed that the rate of production of the organic pigments (reaction 3 ) is linearly related to the photon pressure difference $\mathrm{P}_{\mathrm{I}}-\mathrm{P}_{\mathrm{P}}$. This pressure difference is essentially related to the free energy available for the formation of the pigment. If the photon pressure $\mathrm{P}_{\mathrm{P}}$ associated with the newly formed pigments is low, then more photon energy will flow into the production process, pigments will be produced at a greater rate.

In terms of the affinities and flows of the different processes, we can write Prigogine's general evolutionary criterion, Eq. (4) once arriving at the stationary state, in the following form,

$$
d_{X} P=d P-d_{J} P=d\left(\sum_{k} \frac{A_{k}}{T} v_{k}\right)-\sum_{k} \frac{A_{k}}{T} d v_{k}=0
$$

where the sum is over the three photon dissipative processes, $A_{1}$ is the affinity for the conversion of energy of the solar photon spectrum into energy of the vibrationally excited intermediate precursor molecule photon spectrum, $A_{2}$ is the affinity for the conversion of energy of the excited intermediate precursor molecule photon spectrum into energy of the emitted photon spectrum in the infrared, and $A_{3}$ is the affinity for the photochemical reaction producing the organic pigment.

For the case of equilibrium photon distributions (black-body spectra), the affinities will go as the logarithm of the ratio of the photon pressures [88],

$$
\mathrm{A}_{1}=\mathrm{k}_{\mathrm{B}} \mathrm{T}_{\mathrm{I}} \log \left(\frac{\mathrm{P}_{\mathrm{S}}}{\mathrm{P}_{\mathrm{I}}}\right), \mathrm{A}_{2}=\mathrm{k}_{\mathrm{B}} \mathrm{T}_{\mathrm{E}} \log \left(\frac{\mathrm{P}_{\mathrm{I}}}{\mathrm{P}_{\mathrm{E}}}\right), \mathrm{A}_{3}=\mathrm{k}_{\mathrm{B}} \mathrm{T}_{\mathrm{P}} \log \left(\frac{\mathrm{P}_{\mathrm{I}}}{\mathrm{P}_{\mathrm{P}}}\right)
$$

The ${ }^{v} \mathrm{~K}$ in Eq. (7) are the rates of the corresponding energy conversion (dissipation) processes which we assume are related to the differences of the photon pressures (see discussion at the beginning of this section) attributed to the different black-body spectra.

Since the organic pigments are assumed to act as catalysts for the conversion of energy from the stellar spectrum to the emitted spectrum (i.e., the local heat of photon dissipation by the pigment can be dissipated into the environment (reaction 1+2) or can act as a catalyst for the photochemical production of a new pigment (reaction 3 )), and since $\mathrm{Pp}$ is related to the concentration of the pigments, then we could model the autocatalytic process by multiplying the first dissipation process, $P_{S} \rightarrow P_{I}$, by a factor $(1+\alpha \mathrm{Pp})$ where $\alpha$ represents the effectiveness of the organic pigment to act as a catalyst for photon energy conversion to heat (i.e., $\alpha \rightarrow \infty$ for an excellent catalyst, and $\alpha \rightarrow 0$ for a completely ineffective catalyst). Therefore, the rates of conversion, assuming all constants of proportionality equal to one for convenience (again, we are only interested in showing qualitatively the nonequilibrium dynamics of pigment proliferation), are given by

$$
\mathrm{v}_{1}=\left(1+\alpha \mathrm{P}_{\mathrm{P}}\right)\left(\mathrm{P}_{\mathrm{S}}-\mathrm{P}_{\mathrm{I}}\right) \mathrm{v}_{2}=\left(\mathrm{P}_{\mathrm{I}}-\mathrm{P}_{\mathrm{E}}\right) \mathrm{v}_{3}=\left(\mathrm{P}_{\mathrm{I}}-\mathrm{P}_{\mathrm{P}}\right)
$$

Note the non-linear relation between the forces, Eq. (8), and flows, Eq. (9).
Using Eq. (7) for the steady state together with Eqs. (8) and (9), taking the Boltzmann constant $k_{\mathrm{B}}=1$ for convenience, and observing that the free forces can be characterized in terms of the two free pressures, $\mathrm{P}_{\mathrm{I}}$ and $\mathrm{P}_{\mathrm{p}}$ (since $\mathrm{P}_{\mathrm{S}}$ and $\mathrm{P}_{\mathrm{E}}$ are fixed and given by the local star surface temperature to the fourth power and the infrared emission temperature to the fourth power respectively) gives:

$$
\begin{aligned}
& \frac{\partial}{\partial P_{I}}\left[\left(1+\alpha P_{P}\right)\left(P_{S}-P_{I}\right) \log \frac{P_{S}}{P_{I}}+\left(P_{I}-P_{E}\right) \log \frac{P_{I}}{P_{E}}+\left(P_{I}-P_{P}\right) \log \frac{P_{I}}{P_{P}}\right] \\
& +\left(1+\alpha P_{P}\right) \log \frac{P_{S}}{P_{I}}-\log \frac{P_{I}}{P_{E}}-\log \frac{P_{I}}{P_{P}}=0
\end{aligned}
$$

and

$$
\begin{aligned}
& \frac{\partial}{\partial P_{P}}\left[\left(1+\alpha P_{P}\right)\left(P_{S}-P_{I}\right) \log \frac{P_{S}}{P_{I}}+\left(P_{I}-P_{E}\right) \log \frac{P_{I}}{P_{E}}+\left(P_{I}-P_{P}\right) \log \frac{P_{I}}{P_{P}}\right] \\
& -\alpha\left(P_{S}-P_{I}\right) \log \frac{P_{S}}{P_{I}}+\log \frac{P_{I}}{P_{P}}=0
\end{aligned}
$$

At the steady state, we have (Eq. (4)):

$$
d_{X} P=\sum_{k} v_{k} d A_{k}=0
$$

Which, since $\mathrm{A} 1+\mathrm{A} 2$, the overall affinity is a constant, giving $\delta A_{1}+\delta A_{2}=0$, gives, as the only solution to (12),

$$
v_{1}=v_{2}, v_{3}=0
$$

Which implies that at the steady sate no free energy is being diverted into further pigment production $v_{3}=0$. Any mechanism of pigment dispersal, however, would imply a continual production.

Also, at the steady state, solving Eqs. (10) and (11) gives,

$$
\begin{aligned}
& P_{P}=P_{I}=\frac{1}{2 \alpha}\left[\alpha P_{S}-2+\left[4+4 \alpha P_{S}(1-\gamma)+\alpha^{2} P_{S}{ }^{2}\right]^{1 / 2}\right] \\
& \rightarrow(1 / 2)\left(P_{S}+P_{E}\right) \text { for } \alpha \rightarrow 0 \\
& \rightarrow P_{S} \text { for } \alpha \rightarrow \infty
\end{aligned}
$$

where we define $(1-\gamma)=P_{S} / P_{E}(\gamma$ is, therefore, a measure of the "distance" from equilibrium of the system). Therefore, since $P_{S}$ is much greater than $P_{E}$ (the pressures go as the temperature to the fourth power for black-body spectra) equation (14) indicates that the photon pressure related to the organic pigments $P_{P}$, or in other words the pigment concentration, or the free energy which has gone into pigment production, has increased due to its catalytic activity in forming new pigments and dissipating the stellar photon spectrum into the emitted spectrum.

The entropy production of the energy conversion processes, including catalytic activity of the organic pigment, is given by flows times forces, 


$$
\begin{aligned}
& \frac{d_{i} S}{d t}=\sum_{k} v_{k} \frac{A_{k}}{T_{k}}=\left(P_{S}-P_{I}\right)\left(1+\alpha P_{P}\right) \log \frac{P_{S}}{P_{I}}+\left(P_{I}-P_{E}\right) \log \frac{P_{I}}{P_{E}} \\
& +\left(P_{I}-P_{P}\right) \log \frac{P_{I}}{P_{P}}
\end{aligned}
$$

It can also be shown that the entropy production at the stationary state shifts to larger values as a result of the catalytic activity (Prigogine [85], for the corresponding case of chemical reactions).

These results give the non-linear irreversible thermodynamic explanation for the proliferation of organic pigments in the cosmos. Their concentration can become much greater than that expected under near equilibrium conditions due to their catalytic nature in dissipating the stellar spectrum, depending on the ratio of $P_{S} / P_{E}=\left(\frac{T_{S}}{T_{E}}\right)^{4}$ which is a measure of the distance of the system from equilibrium.

In referring to purely chemical reactions, Prigogine [85], in fact, noticed that such a result sheds light on the problem of the occurrence of complicated biological molecules in steady state concentrations which are of orders of magnitude larger than the equilibrium concentrations. In his 1967 book "Thermodynamics of Irreversible Processes" [85] he states, "Thus, for systems sufficiently far from equilibrium, kinetic factors (like catalytic activity) may compensate for thermodynamic improbability and thus lead to an enormous amplification of the steady state concentrations of the catalyst. Note that this is a strictly non-equilibrium effect. Near equilibrium, catalytic action would not be able to shift in an appreciable way the position of the steady state."

\section{Summary and Discussion}

The cosmos is replete with organic pigments which absorb at the UV wavelengths of prominence of the local star and dissipate these high energy photons into the infrared. The low probability of formation of these pigments under the low temperature and high vacuum conditions of space suggest that they are the result of nonequilibrium thermodynamic processes involving photochemical reactions. In this article, we have shown how these pigments should be considered as microscopic dissipative structures formed as a nonequilibrium thermodynamic response to dissipate the impressed stellar photon potential.

The proliferation of the pigments to concentrations many orders of magnitude greater than what could be expected under near equilibrium conditions can be explained within this non-equilibrium thermodynamic framework by invoking the autocatalytic nature of the pigments in dissipating the same photon potential that produced them.

Evidence in favor of our analysis is many fold:

1. Organic pigment ubiquity in interstellar space would not be expected under near equilibrium conditions.

2. Aromatic pigments are found to be more prevalent under environmental light conditions of higher energy UV photons, whereas aliphatic chain pigments are found under conditions of lower energy UV photons. That is, there is a direct relation between the UV flux from a local star and the energy of maximum absorption of the produced pigment (aromatic pigments absorb higher energy photons than aliphatic pigments).
3. The aggregation of the organic pigment molecules into MAON nano-sized structures can be explained thermodynamically on the greater dissipation provided by these structures with many more microscopic degrees of freedom for distributing the vibrational energy as compared to the isolated PAHs.

4. Proliferation of pigments by the same mechanism on Earth has led to the proliferation of the fundamental molecules of life, almost all of which dissipate in the UV-C range, exactly over a UV-C window which existed in Earth's atmosphere during the Archean $[66,68]$.

It may be that in regions of the cosmos, dissipative self-organization of material under stellar photon fluxes has evolved to the point where the emitted spectrum is so far towards the infrared such that the emission lines have become many and too weak to be detectable. It may thus be possible that some of this dissipative structured material is contributing to the baryonic dark matter. Dark matter has been determined to make up some $27 \%$ of the mass-energy density of the universe and baryonic dark matter makes up approximately $10 \%$ of this dark matter density if the universe is at the critical density for closure, and if the standard model for big bang nucleosynthesis is correct. Since the known baryonic matter makes up less than $5 \%$ of the mass-energy density [89], the amount of material structuring dissipatively into the type of baryonic dark matter suggested here could exceed $50 \%$ of all observable matter in the universe. Other explanations for the invisible baryonic dark matter such as supermassive black holes or MACHOS have difficulty accounting for all of the missing mass.

If such self-organization into microscopic photon dissipative structures is indeed occurring in the cosmos, then it could be expected that the fundamental molecules of life (those found in all three domains of life), which appear to have been self-organized dissipative structures in the UV-C [68], should be ubiquitous throughout the cosmos. This would imply that the origin of life, as we know it $[65,90]$ based on these molecules, should also be very common throughout the universe. Other forms of biotic-abiotic biospheres, such as the organic molecules floating in the clouds of Jupiter, absorbing sunlight and channeling the heat of dissipation into the multiple vortices and storms of the Jovian atmosphere, or the organic molecules in the methane lakes of Titan fomenting the methane rain cycle, could be equally, or even more, common.

Finally, we end this paper with an extraordinary quote by Chandra Wickramasinghe on work he performed with Fred Hoyle in the 1970's and 1980's, from his book entitled "A Journey with Fred Hoyle" [91];

"A discovery of a $3.28 \mu \mathrm{m}$ emission feature in the diffuse radiation emitted by the Galaxy confirmed that aromatic molecules of some kind were exceedingly common on a galactic scale. We argued that the infrared emission, not only at $3.28 \mu \mathrm{m}$, but over discrete set of wavelengths: $3.28,6.2,7.7,8.6,11.3 \mu \mathrm{m}$ must arise from the ultraviolet absorption of starlight by the same molecular system that degrades this energy into the infrared. We had shown much earlier that the $2175 \AA$ extinction of starlight may be due to biological organic molecules, and it seems natural then to connect the two phenomena. Thus we developed a unified theory for the infrared emission and ultraviolet extinction by the same ensemble of aromatic molecules."

Wickramasinghe and Hoyle, therefore, very early on, characterized very well the fact that the same aromatic organic molecules were responsible for the UV absorption and infrared degraded emission spectra. Here we hope we have been clear in bringing out the 
thermodynamic significance of this discovery; that the "exceedingly common on a galactic scale" nature of these aromatic molecules is directly related to their ability to "degrade this energy into the infrared".

\section{Acknowledgements}

K.M. would like to thank DGAPA-UNAM project number IN-102316 for financial support.

\section{References}

1. Dunham TJ Jr (1937) Interstellar neutral potassium and neutral calcium. Publ Astron SocPac 49: 26-28.

2. Swings P, Rosenfeld L (1937) Considerations regarding interstellar molecules. Astron J 86 483-486.

3. Cheung AC, Rank DM, Townes CH, Thornton DD, Welch WJ (1968) Detection of NH3 molecules in the interstellar medium by their microwave emission. Phys Rev Lett 21: 1701-1705.

4. Cheung AC, Rank DM, Thornton DD, Welch WJ (1969) Detection of water in interstellar regions by its microwave radiation. Nature 221: 626-628.

5. Snyder LE, Buhl D, Zuckerman B, Palmer P (1969) Microwave detection of interstellar formaldehyde. Phys Rev Lett 22: 679-681.

6. Ball JA, Gottlieb CA, Lilley AE, Radford HE (1970) Detection of methyl alcohol in Sagittarius. Astrophys J 162: L203-L210.

7. Wilson RW, Jefferts KB, Penzias AA (1970) Carbon Monoxide in the Orion Nebula. Astrophys J 161: L43.

8. Snyder LE, Buhl D (1971) Observations of radio emission from interstellar hydrogen cyanide. Astrophys J 163: L47-L52.

9. Kaifu N, Morimoto M, Nagane K, Akabane K, Iguchi T, et al. (1974) Detection of interstellar methylamine. Astrophys J 191: L135-L137.

10. Turner BE, Kislyakov AG, Liszt HS, Kaifu N (1975) Microwave detection of interstellar cyanamide. Astrophys J 201: L149-L152.

11. Oró J (1960) Synthesis of adenine from ammonium cyanide. Biochem Biophys Res Comm 2: 407-412.

12. Oró J and Kimball AP (1961) Synthesis of purines under possible primitive earth conditions. I. Adenine from hydrogen cyanide. Arch Biochem Biophys 94: 217-227.

13. Sanchez RA, Ferris JP, Orgel LE (1967) Studies in prebiotic synthesis. II. synthesis of purin precursors and amino acids from aqueous hydrogen cyanide. J Mol Bio 30: 223-253.

14. Ferris JP, Sanchez RA, Orgel LE (1968) Studies in prebiotic synthesis. III. synthesis of pyrimidines from cyanoacetylene and cyanate. J Mol Bio 33: 693-704.

15. Stocks PG, Schwartz AW (1981) Nitrogen-heterocyclic compounds in meteorites: significance and mechanisms of formations. Geochim Cosmochim Acta 45: 563-569.

16. Callahan MP, Smith KE, Cleaves HJ, Ruzicka J, Stern JC, et al. (2011) Carbonaceous meteorite contain a wide range of extraterrestrial nucleobases. P Natl Acad Sci 108: 13995-13998.

17. Lacy JH, Evans NJ II, Achtermann JM, Bruce DE, Arens JF, et al. (1989) Discovery of interstellar acetylene. Astrophys J.

18. Cernicharo J, Heras AM, Tielens AGGM, Pardo JR, Herpin F, et al. (2001) Infrared Space Observatory's Discovery of C4H2, C6H2, and Benzene in CRL 618. Astrophys J 546: L123-L126.

19. Kwok S (2016) Complex organics in space from Solar System to distant galaxies. Astron Astrophys Rev 24: 8 .

20. McGuire BA, Carroll PB, Loomis RA, Finneran IA, Jewell PR, et al. (2016) Discovery of the interstellar chira molecule propylene oxide $\left(\mathrm{CH}_{3} \mathrm{CHCH}_{2} \mathrm{O}\right)$. Science 352: 1449-1452.

21. Hearnshaw JB (2014) The Analysis of Starlight: Two Centuries of Astronomical Spectroscopy (2nd edn.), Cambridge University Press, NY, USA.

22. Clayton D (2003) Handbook of Isotopes in the Cosmos: Hydrogen to Gallium (1st edn.), Cambridge Planetary Science, Cambridge University Press, UK.

23. Hoyle F, Wickramasinghe NC (1979) On the nature of interstellar grains. Astrophys Space Sci 66 77-90.

24. Pendleton YJ, Allamandola LJ (2002) The Organic Refractory Material in the Diffuse Interstellar Medium: Mid-Infrared Spectroscopic Constraints. Astrophys J Suppl Series 138: 75-98.

25. Witt AN, Boroson TA (1990) Spectroscopy of extended red emission in reflection nebulae. Astrophys J 355: 182-189.
26. Hoyle F and Wickramasinghe NC (1999) Biofluorescence and the extended red emission in astrophysical sources. Astrophys Space Sci 268: 321-325.

27. Kwok S (2012) Organic Matter in the Universe (1st edn.), Wiley-VCH Verlag GmbH \& Co. KGaA Weinheim Germany, pp: 127-141.

28. Allamandola LJ, Tielens AGGM, Barker JR (1985) Polycyclic aromatic hydrocarbons and the unidentified infrared emission bands: auto exhaust along the Milky Way! Astrophys J 290: L25L28.

29. Allamandola LJ, Tielens AGGM, Barker JR (1989) Interstellar polycyclic aromatic hydrocarbonsThe infrared emission bands, the excitation/emission mechanism, and the astrophysical implications. Astrophys J 71: 733-775.

30. Kwok S, Zhang Y (2011) Mixed aromatic-aliphatic organic nanoparticles as carriers of unidentified infrared emission features. Nature 479: 80-83.

31. Kwok S, Zhang Y (2013) Unidentified Infrared Emission Bands: PAHs or MAONs? Astrophys 771: 5 .

32. Papoular R, Conard J, Giuliano M, Kister J, Mille G (1989) A coal model for the carriers of the unidentified IR bands. Astron Astrophys 217: 204-208.

33. Kerridge JF (1999) Formation and processing of organics in the early Solar System. Space Sci Rev 90: 275-288.

34. Gillett FC, Forrest WJ, Merrill KM (1973) 8-13-micron spectra of NGC 7027, BD $+30^{\circ} 3639$, an NGC 6572. Astrophys J 183: 87-93.

35. Russell RW, Soifer BT, Willner SP (1977) The 4 to 8 micron spectrum of NGC 7027. Astrophys J 217: L149-L153.

36. Russell RW, Soifer BT, Willner SP (1978) The infrared spectra of CRL 618 and HD 44179 (CRL 915). Astrophys J 220: 568-572.

37. Knacke RF (1977) Carbonaceous compounds in interstellar dust. Nature 269: 132-134.

38. Duley WW, Williams DA (1981) The infrared spectrum of interstellar dust: surface functional groups on carbon. Mon Not R Astron Soc 196: 269-274.

39. Tielens AGGM (2005) The Physics and Chemistry of the Interstellar Medium (1st edn.), Cambridge University Press, NY, USA, p: 212

40. Mattila K, Lehtinen K, Lemke D (1999) Detection of widely distributed UIR band emission in the disk of NGC 891. Astron Astrophys 342: 643-654.

41. Smith JDT, Draine BT, Dale DA, Moustakas J, Kennicutt Jr RC (2007) The mid-infrared spectrum of star-forming galaxies: global properties of polycyclic aromatic hydrocarbon emission. Astrophys J 656: 770-791.

42. Groves B, Dopita MA, Sutherland RS, Kewley LJ, Fischera J, et al. (2008) Modeling the panspectral energy distribution of starburst galaxies. IV. The controlling parameters of the starburst SED. Astrophys J Supplement Series 176: 438-456.

43. Kwok S (2009) Organic matter in space: from star dust to the Solar System. Astrophys Space Sci 319: 5-21.

44. Keller LP, Bajt S, Baratta GA, Borg J, Bradley JP, et al. (2006) Infrared spectroscopy of comet 81P/ Wild 2 samples returned by Stardust. Science 314: 1728-1731.

45. López-Puertas M, Dinelli BM, Adriani A, Funke B, García-Comas M, et al. (2013) Large Abundances of Polycyclic Aromatic Hydrocarbons in Titan's Upper Atmosphere. Astrophys J 770: 132 .

46. Henning T, Salama F (1998) Carbon in the Universe. Science 282: 2204-2210.

47. Leger A, Puget JL (1984) Identification of the 'unidentified' IR emission features of interstellar dust? Astron Astrophys 137: L5-L8.

48. Puget JL, Leger A (1989) A new component of the interstellar matter: small grains and large aromatic molecules. Ann Rev Astron Astrophys 27: 161-198.

49. Tielens AGGM (2008) Interstellar polycyclic aromatic hydrocarbon molecules. Ann Rev Astron Astrophys 46: 289-337.

50. Uchida KI, Sellgren K, Werner M (1998) Do the Infrared Emission Features Need Ultraviolet Excitation? Astrophys J Lett 493: L109.

51. Uchida KI, Sellgren K, Werner MW, Houdashelt ML (2000) Infrared Space Observatory midinfrared spectra of reflection nebulae. Astrophys J 530: 817-833.

52. Ehrenfreund P, Robert F, d'Hendencourt L, Behar F (1991) Comparison of interstellar and meteoric organic matter at $3.4 \mu \mathrm{m}$. Astron Astrophys 252: 712-717.

53. Kwok S, Volk K, Bernath P (2001) On the Origin of Infrared Plateau Features in Proto-Planetary Nebulae. Astrophys J Lett 554: L87-L90.

54. Guillois O, Nenner I, Papoular R, Reynaud C (1996) Coal Models for the Infrared Emission Spectra of Proto-Planetary Nebulae. Astrophys J 464: 810.

55. Papoular R (2001) The use of kerogen data in understanding the properties and evolution of interstellar carbonaceous dust. Astron Astrophys 378: 597-607. 
Citation: Michaelian K, Simeonov A (2017) Thermodynamic Explanation for the Cosmic Ubiquity of Organic Pigments. Astrobiol Outreach 5: 156. doi:10.4172/2332-2519.1000156

Page 9 of 9

56. Derenne S, Robert F (2010) Model of molecular structure of the insoluble organic matter isolated from Murchison meteorite. Meteorit Planet Sci 45: 1461-1475.

57. Cronin JR, Pizzarello S, Frye JS (1987) 13C NMR spectroscopy of the insoluble carbon of carbonaceous chondrites. Geochim Cosmochim Acta 51: 299-303.

58. Sephton MA (2002) Organic compounds in carbonaceous meteorites. Nat Prod Rep 19: 292-311.

59. Cody GD, Alexander CMO'D (2005) NMR studies of chemical structural variation of insoluble organic matter from different carbonaceous chondrite groups. Geochim Cosmochim Acta 69: 1085-1097.

60. Nakamura-Messenger K, Messenger S, Keller LP, Clemett SJ, Zolensky ME (2006) Organic globules in the Tagish Lake meteorite: remnants of the protosolar disk. Science 314: 1439-1442.

61. Martins Z, Botta O, Fogel ML, Sephton MA, Glavin DP, et al. (2008) Extraterrestrial nucleobases in the Murchison meteorite. Earth Planetary Sci Lett 270: 130-136.

62. Kwok S (2007) Molecules and solids in planetary nebulae and proto-planetary nebulae. Adv Space Res 40: 655-658.

63. Joblin C, Tielens AGGM, Allamandola LJ, Geballe TR (1996) Spatial Variation of the 3.29 and 3.40 Micron Emission Bands within Reflection Nebulae and the Photochemical Evolution of Methylated Polycyclic Aromatic Hydrocarbons. Astrophys J 458: 610.

64. Sloan GC, Jura M, Duley WW, Kraemer KE, Bernard-Salas J, et al. (2007) The unusua hydrocarbon emission from the early carbon star HD 100764: the connection between aromatics and aliphatics. Astrophys J 664: 1144-1153.

65. Michaelian K (2011) Thermodynamic dissipation theory for the origin of life. Earth Syst Dyn 2 37-51.

66. Michaelian K (2013) A non-linear irreversible thermodynamic perspective on organic pigment proliferation and biological evolution. J Phys Conf Series 475: 012010.

67. Michaelian K, Simeonov A (2014) Fundamental molecules of life are pigments which arose and evolved to dissipate the solar spectrum.

68. Michaelian K, Simeonov A (2015) Fundamental molecules of life are pigments which arose and co-evolved as a response to the thermodynamic imperative of dissipating the prevailing solar spectrum. Biogeosciences 12: 4913-4937.

69. Vekshin NL (2002) Photonics of Biopolymers (2nd edn.), Springer, Berlin, Heidelberg.

70. Gradie J, Veverka J (1980) The composition of the Trojan asteroids. Nature 283: 840-842.

71. Roush TL, Cruikshank DP (2004) Observations and laboratory data of planetary organics. In: Ehrenfreund P, et al. (eds.), Astrobiology: Future Perspectives, Chapt. 7, pp: 149-177.

72. Waite JH Jr (2007) The process of tholin formation in Titan's upper atmosphere. Science 316 870-875.
73. Nguyen MJ, Raulin F, Coll P, Derenne S, Szopa C, et al. (2007) Carbon isotopic enrichment in Titan's tholins? implications for Titan's aerosols. Planet Space Sci 55: 2010-2014.

74. Atreyaa SK, Adamsa EY, Niemann HB, Demick-Montelara JE, Owen TC, et al. (2006) Titan's methane cycle. Planet Space Sci 54: 1177

75. Clark RN, Brown RH, Jaumann R, Cruikshank DP, Nelson RM, et al. (2005) Compositional maps of Saturn's moon Phoebe from imaging spectroscopy. Nature 435: 66-69.

76. Cruikshank DP, Dalton JB, Dalle Ore CM, Bauer J, Stephan K, et al. (2007) Surface composition of Hyperion. Nature 448: 54-56.

77. Cruikshank DP, Wegryn E, Dalle Ore CM, Brown RH, Bibring JP, et al. (2008) Hydrocarbons on Saturn's satellites Iapetus and Phoebe. Icarus 193: 334-343.

78. Denk T, Neukum G, Roatsch T, Porco CC, Burns JA, et al. (2010) Iapetus: unique surface properties and a global color dichotomy from Cassini imaging. Science 327: 435-439.

79. Spencer JR, Denk T (2010) Formation of Iapetus' Extreme Albedo Dichotomy by Exogenically Triggered Thermal Ice Migration. Science 327: 432-435

80. Cruikshank DP, Dalle Ore CM, Clark RN, Pendleton YJ (2014) Aromatic and aliphatic organic materials on Iapetus: Analysis of Cassini VIMS data. Icarus 233: 306-315.

81. Jewitt DC, Luu JX (2001) Colors and spectra of Kuiper Belt objects. Astron J 122: 2099-2114.

82. Mumma MJ, Charnley SB (2011) The Chemical Composition of Comets-Emerging Taxonomies and Natal Heritage. Annual Rev Astron Astrophys 49: 471-524.

83. Whipple FL (1978) On the Nature and Origin of Comets and their Contribution to Planets. The Moon and Planets 19: 305-315.

84. Clemett SJ, Sanford SA, Nakamura-Messenger K, Hörz F, McKay DS (2010) Complex aromatic hydrocarbons in Stardust samples collected from comet 81P/Wild 2. Meteorit Planet Sci 45: $701-722$

85. Prigogine I (1967) An Introduction to the Thermodynamics of Irreversible Processes (1st edn.), Wiley, NY, USA

86. http://www-oc.chemie.uni-regensburg.de/OCP/ch/chb/oc5/Photochemie-08.pdf

87. Glansdorff P, Prigogine I (1964) On a general evolution criterion in macroscopic physics. Physica 30: $351-374$.

88. Herrmann F, Würfel P (2005) Light with nonzero chemical potential. Am J Phys 73: 717-721.

89. http://science.nasa.gov/astrophysics/focus-areas/what-is-dark-energy/

90. Michaelian K (2009) Thermodynamic origin of life.

91. Wickramasinghe C (2013) A Journey with Fred Hoyle (2nd edn.). In: Wickramasinghe K (ed.) World Scientific Publishing Co. 